Virginia Dignum: An Overview of Agents in Knowledge Management. In: Proceedings of INAP-05, M. Umeda et al. (Eds), LNAI-4369, Springer, 2006, pp. 175-189. 


\title{
An Overview of Agents in Knowledge Management
}

\author{
Virginia Dignum \\ Universiteit Utrecht \\ P.O. Box 80089,3508 TB Utrecht \\ The Netherlands \\ virginia@C.s.uu.nI
}

\begin{abstract}
Current developments in Knowledge Management concern the sharing and usage of knowledge in dynamic environments. The need for systems that both react to and anticipate the needs and expectations of users calls for flexible and adaptable development and implementation frameworks. These are exactly the characteristics that identify software agents and agent societies, which make natural the application of the agent paradigm in KM. This paper attempts to identify both the advantages of agents for KM, and the aspects of KM that can benefit most from this paradigm. Furthermore, the paper describes several current KM projects that use agent technology and identifies open research areas
\end{abstract}

\section{Introduction}

The main goal of Knowledge Management (KM) is to provide relevant knowledge to assist users in executing knowledge intensive tasks. $\mathrm{KM}$ is about facilitating an environment where work critical information can be created, structured, shared, distributed and used. To be effective such environments must provide users with relevant knowledge, that is, knowledge that enables users to better perform their tasks, at the right time and in the right form. Knowledge Management (KM) has been a predominant trend in business in the recent years. Scaling-up research prototypes to real-world solutions usually requires an application-driven integration of several basic technologies, e.g., ontologies for knowledge sharing and reuse, collaboration support like CSCW systems, and personalized information services.

As it is often mentioned in the literature, knowledge tasks have a collaborative aspect, that is, an individual can best acquire and use knowledge by reusing information already collected and annotated by others or by making use of existing relations among people (communities) [9]. Furthermore, a KM system must be able to adapt to changes in the environment, to the different needs and preferences of users, and to integrate naturally with existing work methods, tools and processes. That is, KM systems must be reactive (able respond to user requests or environment changes) and proactive (able to take initiatives to attend to user needs). These aspects also characterise intelligent software agents, what seems to indicate the applicability of agent technology in the KM area.

Intelligent agents are a relatively new paradigm for developing software applications and are currently the focus of intense interest on the part of many fields 
of computer science and artificial intelligence [7]. Agents have made it possible to support the representation, co-ordination, and co-operation between heterogeneous processes and their users. Internet technology and software agents make thus possible to build sophisticated, well performing KM systems designed to deliver content, from multiple, heterogeneous sources, to each individual, in the individual's specific context and under the individual's own control. This ability contributes to improve the relationship between knowledge suppliers and consumers by providing both parties more precise control over the interaction.

In order to cope with the inherent complexity of a more comprehensive solution, the concept of Agent-mediated Knowledge Management (AMKM) proposes agentbased approaches to deal with collective aspects of the domain in an attempt to cope with the conflict between desired order and actual behavior in dynamic environments [4]. Inherent to AMKM is a social layer, which structures the society of agents by defining specific roles and possible interactions between them.

In this paper, we intend to show the applicability of agent technology to the development of collaborative KM environments that address the problems highlighted above. The paper is organised as follows: Section 2 provides some generic background on Knowledge Management and software agents. Section 3 discusses the applicability potential of agents to the KM area. In section 4, some current KM projects that make use of agent technology are listed. In section 5, discusses issues related to AMKM design and methodology, on the basis of the OperA framework being developed at our institute. Finally, section 6 presents our conclusions and discusses some open issues and highlights aspects for further research.

\section{Background}

\subsection{Knowledge Management Environments}

Business processes are often highly dynamic and unpredictable. This makes it difficult to give a complete a priori specification of all the activities that need to be performed, which their knowledge needs are, and how those activities should be ordered. Within organisations, there is a decentralised ownership of the tasks, information and resources involved in the business process. Different groups within organisations are relatively autonomous, in the sense that they control how their resources are created, managed or consumed, and by whom, at what cost, and in what period [6]. Furthermore, often multiple and physically distributed organisations (or parts hereof) are involved in the business process. Each organisation attempts to maximise its own profit within the overall activity. That is, there is a high degree of natural concurrency (many interrelated tasks and actors are active at any given point of the business process) which makes important to monitor and manage the overall business process (e.g. total time, total budget, etc.). These characteristics call for an environment that integrates the business process aspects of knowledge work with active support for searching, using and adding heterogeneous knowledge sources [15].

The first attempts towards business integration were made at the data level, where distributed computing frameworks have been developed to support distributed 
computing in heterogeneous environments and provide an interface description language and services that allow objects to be defined, located and invoked across locations and applications. However, knowledge assets available in an organisation are more than data sources alone. Such assets include structured and unstructured information, multimedia knowledge representations and links to people (ex. through knowledge maps or yellow pages). Besides using existing knowledge sources, the environment should be able to create (and store) new knowledge based on its observation of the user's task performance [11]. Furthermore, there is a need to combine formal and informal knowledge representations, as well as heterogeneous multimedia knowledge sources. At knowledge level, integration must be based on the semantics and the context of the problem at hand. A knowledge-level integration framework must be able to create dynamic relationships between knowledge-intensive business processes and knowledge sources that do not compromise the autonomy of the different parts. In order to be able to support the execution of knowledge-intensive tasks, using knowledge from heterogeneous sources, according to diverse user preferences, KM system must be able to provide a common knowledge description. In this way, integration and autonomy are achieved by separating the use of knowledge from the specific characteristics of the knowledge source. KM systems must therefore provide uniform access to a diversity of knowledge and information sources of different degree of formality. Furthermore, knowledge management environments must be able to adapt to the different needs and preferences of users, and integrate naturally with existing work methods, tools and processes. That is, such environments must be reactive and proactive.

\subsection{Intelligent Software Agents}

The key issue in software agents is autonomy, which refers to the principle that agents can operate on their own, without the need for human guidance. An autonomous agent has the control over its own actions and internal state. That is, an agent can decide whether to perform a requested action. Furthermore, agents are problem-solving entities, with well-defined boundaries and interfaces, designed to fulfil a specific purpose and exhibit flexible and pro-active behaviour. Autonomous agents have the possibility to interact with other agents using a specific communication language, thus creating a sort of social ability that allows them to perceive their environment, respond to its changes or achieve goals by simply adapting and reacting to other players. A Multi-Agent System (MAS) can therefore be defined as: "a collection of possibly heterogeneous, computational entities, having their own problem solving capabilities and which are able to interact among them in order to reach an overall goal” [5].

Agents usually operate in a dynamic, non-deterministic complex environment, in which a single input action can often produce unexpected results. MAS environments assume no global control, data decentralization and asynchronous computation. Furthermore, agents in a MAS are assumed to operate with incomplete information or capabilities for solving the problem. Communication is thus the key for agents to share the information they collect, to co-ordinate their actions and to increase interoperation. In heterogeneous systems, knowledge sharing is hampered by the lack of 
common ontologies. Therefore, adequate support for ontology matching and meaning negotiation is of great importance to MAS and to AMKM in special.

\section{Agents in Knowledge Management}

KM environments can be described as distributed system where different actors, acting autonomously on behalf of a user, and each pursuing its own goals, need to interact in order to achieve their goals. In such environments, the ability to communicate and negotiate is paramount. Furthermore, the number and behaviour of participants cannot be fixed a priori and the system can be expected to expand and change during operation, both in number of participants as in amount and kind of knowledge shared. The choice for multi-agent systems for KM is motivated by the following observations:

- KM domains involve an inherent distribution of data, problem solving capabilities and responsibilities (conforms to the ideas of autonomy and social ability of agents).

- The integrity of the existing organisational structure and the autonomy of its subparts need to be maintained (uses autonomous nature of the agents).

- Interactions in KM environments are fairly sophisticated, including negotiation, information sharing, and coordination (requires complex social skills with which agents are endowed).

- KM domains call for a functional separation between knowledge use and knowledge sources as a way to incorporate dynamic behaviour into information systems design (agents can act as mediators between source and application of knowledge).

- The solution for KM problems cannot be entirely prescribed from start to finish and therefore problem solvers are required that can respond to changes in the environment, to react to the unpredictability of business process and to proactively take opportunities when they arise (requires the reactive and proactive abilities of agents).

In order to cope with the inherent complexity of a more comprehensive solution, the concept of Agent-mediated Knowledge Management (AMKM) proposes agentbased approaches to deal with collective aspects of the domain in an attempt to cope with the conflict between desired order and actual behavior in dynamic environments. Inherent to AMKM is a social layer, which structures the society of agents by defining specific roles and possible interactions between them. Van Elst, Dignum and Abecker argued that "the basic features of agents (social ability, autonomy, re- and pro-activeness) can alleviate several of the drawbacks of the centralized technological approaches for KM" [4]. In that article, we proposed three dimensions to describe agent KM systems: the system development layer (from analysis to implementation of the system), the macro-level structure of the system (single agent and Multi-agents models) and the KM applications area (share, learn, use, distribution and so on). Taking into account the second dimension, a classification of software or experimental models of agent systems was proposed that could support KM. For example, Personal Information Management (PIM) tools could be considered as 
single agent systems whose task is to support knowledge workers in their daily work in a way to become "a personal assistant who is collaborating with the user in the same work environment" [13]. Many different examples fall into this category, like Lieberman's Letizia [12] and the OntoBroker developed by Staab and Schnurr [15].

In KM environments, agents can check of the dynamic conditions of the environment, reason to interpret those perceptions, solve problems, draw inferences and determine actions, and finally, act accordingly. The use of agents in KM can be seen in two perspectives. In one hand, agents can be used to model the organisational environment where the KM system will operate and, on the other hand, software agents can be used to implement the functionality of KM systems. Most existing KM projects involving agent technology concentrate on using agents as implementation tool modelling primitives. Agents are used there to support and extend the activity of (human) users. However, more and more interest is arising about the advantages of agent-based modelling of KM environments. In the remaining of this section, we will describe both perspectives in more detail.

\subsection{Agent-based models for $\mathrm{KM}$ environments}

Agent-based models for knowledge management see agents as autonomous entities (like employees in a company) that are endowed with certain behaviours and the interactions among these entities give rise to complex dynamics. In this context, agents can be defined as 'one that acts or has the power or authority to act' or 'one that takes action at the instigation of another'. The concept of agent in this sense is not new, nor restricted to software. In this perspective, agents are autonomous social entities that exhibit flexible, responsive and proactive behaviour.

An organisation can be seen as a set of entities and their interactions, which are regulated by mechanisms of social order and created by more or less autonomous actors to achieve common goals. Business environments must furthermore consider the behaviour of the global system and be able to incorporate collective characteristics of an organisation such as stability over time, some level of predictability, and clear commitment to aims and strategies. While current research often takes individual agents as starting point and looks at interaction from the perspective of an individual agent, that is, how it affects and influences the goals and beliefs of the agent, agent models for organisations must take the perspective of the organisation as a whole. That is, multi-agent systems, or agent societies, must therefore be able to define the global aims as well as the roles and responsibilities of participants.

Agent societies represent the interactions between agents and are as such the virtual counterpart of real-life societies and organisations. Individual agents model specific roles in the society and interact with others as a means to accomplish the goals specified by those goals. This perspective makes the design of the system less complex since it reduces the conceptual distance between the system and the realworld application it has to model. Therefore, agent societies are an effective platform for virtual organisations because they provide mechanisms to allow organisations to advertise their capabilities, negotiate their terms, exchange rich information, and synchronise processes and workflow at a high-level of abstraction [15]. 
From an organisational perspective, the main function of an individual agent is the enactment of a role that contributes to the global aims of the society. That is, society goals determine agent roles and interaction norms. Agents are actors that perform role(s) described by the society design. The agent's own capabilities and aims determine the specific way an agent enacts its role(s). However, the society is often not concerned about which individual agent will actually play a specific role as long it gets performed. Several authors have advocated role-oriented approaches to agent society development, especially when it is manifest to take an organisational view on the application scenario [17], [18].

\subsection{Using agents to implement $\mathrm{KM}$ systems}

Knowledge Management Environments can be implemented as communities of different types of agents that collaborate to provide the required support to users on their knowledge intensive tasks. In agent-based implementations of knowledge management systems, software agents are employed as tools to manage loosely coupled information sources, to provide unifying presentation of distributed heterogeneous components and to personalise knowledge presentation and navigation. Possible agent-based services in a KM system are [8]:

- Search for, acquire, analyse, integrate and archive information from multiple heterogeneous sources,

- Inform us (or our colleagues) when new information of special interest becomes available,

- Negotiate for, purchase and receive information, goods or services,

- Explain the relevance, quality and reliability of that information,

- Learn, adapt and evolve to changing conditions.

These services are often specified in terms of the following types of agents:

- Personal Assistants represent the interests of the user and provide the interface between users and the system. They are concerned with user preferences and needs, and will present information in the preferred format, at the right time. A proactive personal assistant agent will not only perform the tasks given to it by the user, but will also suggest knowledge sources or other resources that are not explicitly requested if they match the user's interests.

- Cooperative Information Agents (CIAs) focus on accessing multiple, distributed and heterogeneous information sources. A CIA needs to maintain actively its information by communicating with others and reasoning about its own information.

- Task analysts are agents that monitor a certain task in the business process, determine the knowledge needs of the task, and gather that knowledge by communicating with other agents. The agent can also monitor the execution of the task and evaluate the applicability of the knowledge provided. The lessons learned here are used to update its internal state and optimising task knowledge.

- Source keepers are agents dedicated to maintaining knowledge sources and are responsible for describing the knowledge contained in the source and extract relevant information for a given request. Source keepers can also actively 
propose uses for its source to other agents based on its own knowledge of other agents' needs.

- Mediators are agents that can provide a number of intermediate information services to other agents. They may suggest collaboration between users with common interests, or provide information about the tools available.

\section{Applications of agents in $\mathrm{KM}$}

Knowledge Management systems typically employ a strong organizational perspective. Macro-level questions, such as number of agents or stakeholders, the typology of information flows and the coordination of decisions are therefore of special concern. One possible way of characterize AMKM systems, as proposed in [Springer] is the degree of sociability of its agents:

- Single-agent architectures: agents interact with its (information) environment and with the user, but have no (elaborated) interaction with other agents, in special, no cooperative action is expected. Typical examples include agentbased user interfaces and PIM agents.

- Closed multi-agent architectures: agents co-operate with other agents in order to solve their tasks. These agents do not necessarily have to have exactly the same goals, but their tasks and capabilities are comparable. Societies are homogenous, and participating agents are known from the beginning. Agentbased collaborative filtering is a typical example for this class of MAS.

- Open multi-agent architectures: contain multiple agent classes which may have completely different purposes, knowledge and capabilities. Specialists exist for wrapping information sources, agents for integrating different description schemas, and for adequately presenting information to the users. Up to a certain extent, agents can enter of leave the society at all times, and represent different stakeholders, with possibly conflicting objectives. All these different agent types have to cooperate and bring in their complementary expertise in order to accomplish the overall goal of the system.

Furthermore, AMKM applications are characterized specifically by the knowledge management functionality they are meant to support. In particular, taking in account the well-known KM cycle, we classify AMKM applications by their focus in terms of KM processes:

- Identification processes analyze what knowledge exists in an organization, what the knowledge containers are, who the stakeholders are, etc.

- Acquisition is the process of integrating external knowledge into an organization.

- Development processes generate new knowledge in the organization.

- Distribution processes connect knowledge containers with potential users.

- Preservation aims at the sustainability of knowledge, i.e., that is accessible and understandable over a period time.

- Utilization means to operationalize available knowledge in order to solve actual business tasks better. 
From these classical models, several further distinctions have been developed in Knowledge Management research that can be utilized to describe the application area. For example, systems can take a more process-oriented or a more product-oriented view [10], [14]. The latter emphasizes the management of explicit knowledge contained in "knowledge products" such as databases, documents, formal knowledge bases etc., whereas the former focuses on human beings and their internal knowledge, i.e., the "process of knowing" and the "process of exchanging knowledge" between people. Typical systems that take a product-oriented view are document retrieval agents. Expert finder systems, on the other hand, take a more process-oriented view. Furthermore, a KM system can support individuals and their tasks at hand, it can support teams and groups, or it may take a more global, organizational perspective. The theoretical analysis of Knowledge Management characteristics in Section 1 may be the source of further possible application areas for information technology, e.g., facilitating trust, motivating users to share knowledge, or establishing group awareness. Concrete agent-based KM applications may deal with one or a few of these aspects, or they may be more comprehensive frameworks that try to cover large parts of the KM cycle. In the following section we will analyze existing agent-based KM applications, illustrative for different approaches.

\section{AMKM Design and Methodology}

Only a few of the existing AMKM systems provide a comprehensive agent-based KM methodology that enables the development of KM support systems in organizational contexts from a software engineering perspective. Agent-Oriented Software Engineering (AOSE) methodologies provide models and methods adequate to represent and support all types of activities throughout all phases of the software lifecycle. AOSE methodologies must be both specific enough to allow engineers to design the system, and generic enough to allow the acceptance and implementation of multi-agent systems within an organization, allowing for the involvement of users, managers and project teams. From an organizational point of view, the behavior of individual agents in a society can only be understood and described in relation to the social structure. Therefore, the engineering of agent societies needs to consider both the interacting and communicating abilities of agents as well as the environment in which agent societies are situated. Furthermore, in open societies the 'control' over the design of participating agents lies outside the scope and design of the society itself. That is, the society cannot rely on the embedding of organizational and normative elements in the intentions, desires and beliefs of participating agents. These considerations lead to the following requirements for engineering methodologies for agent societies [2]:

- Include formalisms for the description, construction and control of the organizational and normative elements of a society (roles, norms and goals).

- Provide mechanisms to describe the environment of the society and the interactions between agents and the society, and to formalize the expected outcome of roles in order to verify the overall animation of the society. 
- The organizational and normative elements of a society must be explicitly specified because an open society cannot rely in its embedding in the agent's internal structure.

- Methods and tools are needed to verify whether the design of an agent society satisfies its design requirements and objectives.

- Provide building directives concerning the communication capability and ability to conform to the expected role behavior of participating agents.

A recent proposal for a AMKM design framework and methodology is OperA [1]. OperA uses the agent paradigm to analyse and model organisations and their knowledge needs, and to provide a reusable architecture to build KM systems. Different knowledge intensive tasks need knowledge from different sources and in different presentation formats. Therefore, the framework distinguishes between application, description and representation of knowledge and provides a common, uniform description of knowledge items (both sources and needs). A community of collaborative agents is responsible for the matching of knowledge supply and demand taking in account the user needs and preferences and the knowledge needs of a task. By collaborating with each other and with users, agents will learn and dynamically extend this framework by checking the current conditions of the environment. Agents will collaborate to interpret those perceptions, solve problems, draw inferences and determine actions, and finally, act accordingly. Information agents specialised in the different types of sources can provide this description.

\subsection{OperA Model and Methodology}

The framework for agent societies we propose models the collective and interaction aspects of the society from an organisational perspective based on the notions of agent, role, norms, communication and goals. We propose a framework for agent societies consisting of three interrelated models each describing different aspects of the society that attempts to cope with the difference between desired order (from an organisational perspective) and actual behaviour (as actually realised by the participants) in dynamic environments [17]:

- The organisational model is the result of the observation and analysis of the domain and describes the desired behaviour of an agent society, as determined by the society 'owners' in terms of goals, rules, roles and interactions.

- The social model maps organisational roles to specific agents. Agreements concerning the role(s) an agent will play and the conditions of the participation are described in social contracts.

- The agent model specifies the interaction agreements between agents as interaction contracts. This model accounts for the actual (emergent) behaviour of the society.

A methodology to analyse a given domain and determine the type and structure of the agent society that best models that domain is described in [3]. Organisation theory shows that different organisations with exhibit different requirements for coordination and interaction. Coordination models are determined by transaction costs and reflect the balance between organisational objectives and activities. For example, the market 
model fits well in an exchange situation, and the hierarchical model can be used in production settings. The methodology provides generic facilitation and interaction frameworks for agent societies that implement the functionality derived from the coordination model applicable to the problem domain. Standard society types as market, hierarchy and network, can be used as starting point for development and can be extended where needed and determine the basic norms and facilitation roles necessary for the society.

\subsection{AMKM architecture}

The AMKM architecture based on the model described in [1][3] consists of two layers: operation and facilitation as depicted in figure 1. At production level, there are basically three types of agents: personal assistants, (business) process task analysts and knowledge source keepers. Depending on the application area, other agent types may be needed what can be determined by the application of the methodology introduced in [3]. Each of those agents provides a transparent access to its organisational background. That is, a personal assistant concentrates on the fulfilment and description of its user needs and does not need to know the type and format of knowledge sources, which are encapsulated by source agents, with whom the personal assistant can communicate.

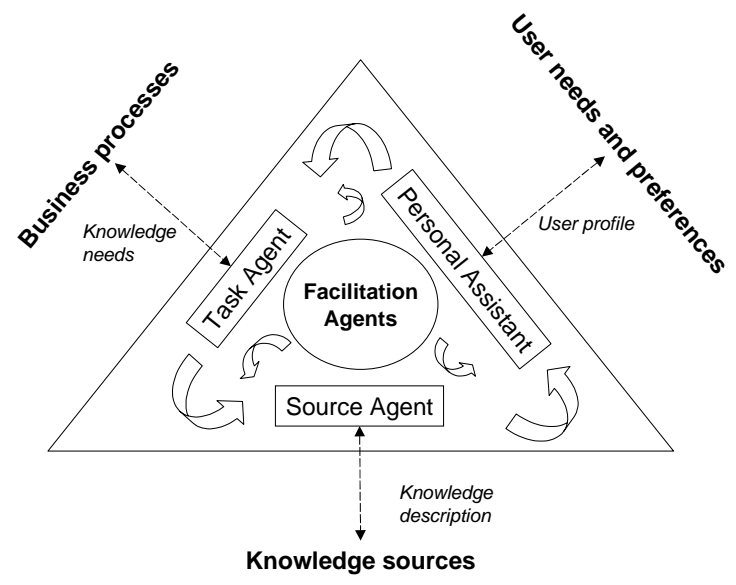

Fig. 1. Generic architecture for Agent-mediated Knowledge Management

The facilitation level helps agents to locate each other, based on their needs and facilities. That is, at facilitation level, the 'norms' of the society are kept and enforced and interaction is ensured. Furthermore, facilitation agents ensure interaction by monitoring and supporting contract formation, take care of introducing new agents to the rules of the society and keep track of the reputation of trading agents. Typical facilitation agent roles are matchmakers, gatekeepers and reputation agents. 


\subsection{An application of AMKM framework}

Based on the OperA Model, we are developing a Knowledge Market to support KennisNet members to exchange knowledge according to the requirements expressed in the previous section. This model enables for the incorporation of individual initiative (embodied in personal agents) within organizational processes (described by organizational model of the society). The model is further described in the following subsections.

\subsubsection{Organizational Model}

The social activity of agents is coordinated at the facilitation level. That is, at facilitation level, the 'norms' of the society are kept and enforced and interaction is ensured. Facilitation agents ensure interaction by monitoring and supporting contract formation, take care of introducing new agents to the rules of the society and keep track of the reputation of trading agents. Typical facilitation agent roles are matchmakers, gatekeepers and reputation agents. Gatekeepers are responsible for accepting and introducing new agents to the knowledge market. Matchmakers keep track of agents in the system, their needs and possibilities and mediate in the matching of demand and supply of knowledge. Notaries register and keep track of collaboration contracts between agents. Finally, monitoring agents are trusted third parties that keep track of the execution of collaboration contracts between agents.

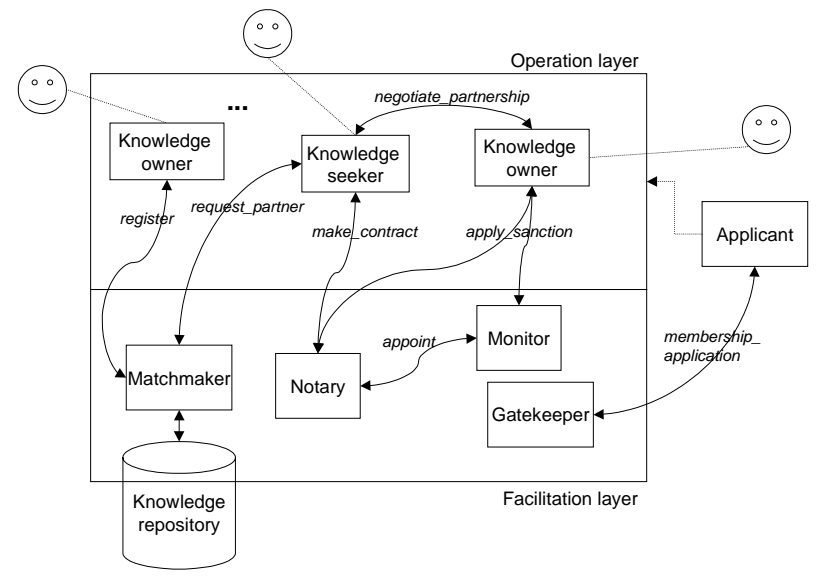

Fig. 2. Knowledge Market architecture

The operational roles identified from the requirements and domain characteristics are knowledge seeker and knowledge owner, which are both specific aspects of personal assistants. The seeker agent provides the interface between the user seeking collaboration and the market and reflects the personal preferences, learning style and work process of the user. Owner agents are responsible to 'advertise' the capabilities of a knowledge worker and vindicate the interests of the knowledge owner. The owner agent can also actively offer the services and skills of its user propose uses for 
its source to other agents based on its own knowledge of other agents needs or indicated by the matchmaker. Figure 1 depicts the roles and role dependencies in the Knowledge Market.

The interaction structure displayed in figure 2 describes the activity of the user roles (knowledge owner and seeker) in the Knowledge Market. Knowledge seekers and knowledge owners apply to enter the society through the 'Member registration' scene. If the application is successful, the agent proceeds can choose to 'request partner', 'publish' some knowledge item of its own, or 'browse' the repository. In the 'request partner' scene, both seeker and owner agents can initiate an exchange by respectively announcing a need or a skill. In the 'negotiate partnership' scene, seeker and owner discuss the conditions of an exchange. The result is an interaction contract that describes an instance of the 'exchange' scene. Interaction scripts serve as a blueprint for the actual interactions between agents enacting roles. That is, an instantiation of a scene script can adapt the script to accommodate specific requirements of the enacting agents. Scene instances must however, comply with script norms and objectives.

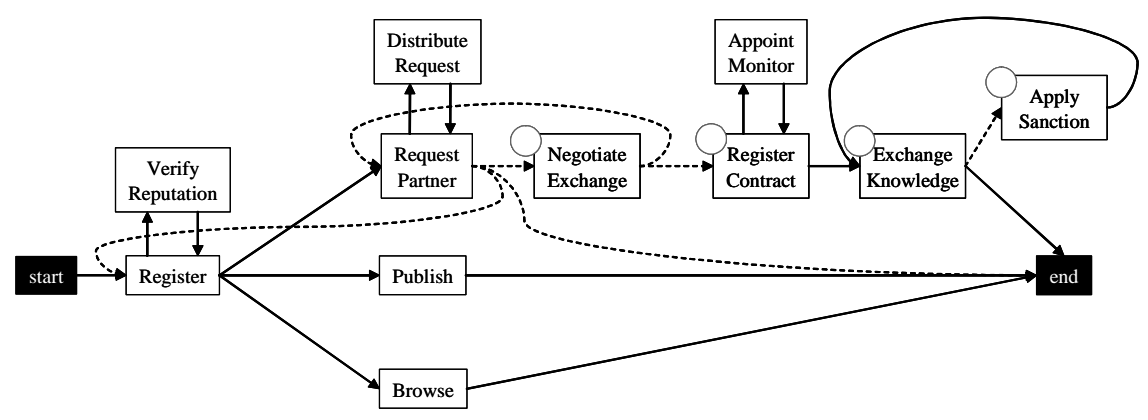

Fig. 3. Interaction structure of Knowledge Market

\subsubsection{Social Model}

Social contracts describe the agreements between participating agents and the Knowledge Market society. People seeking collaboration can initiate through the user interface of the Knowledge Market a personal agent that will act as their avatar in the system. This agent will use the preferences and conditions specified by the user to find appropriate partners and negotiate exchange terms. Furthermore, factors such as privacy, secrecy and competitiveness between brands and departments may influence the channels and possibilities of sharing and must thus be considered. Matching of supply and demand of knowledge is very complex and requires techniques such as fuzzy matching algorithms, or multi-attribute matching ${ }^{1}$. Due to space restrictions we will not further discuss this here.

${ }^{1}$ That is, how much is a specific piece of knowledge worth, at a specific moment, under the specific circumstances holding and to the specific partners involved in the exchange. 
Negotiation of social contracts is done between the applicant agent and the Gatekeeper agent, which will watch over the interests of the society itself. For example, imagine that Anne is a member of the KennisNet group that is seeking knowledge on price policies from the competition. Anne will initiate an agent enacting the knowledge seeker role in the Knowledge Market. During the Member admittance scene, the conditions for Anne's agent will be negotiated and fixed in a social contract. This contract can specify, for instance, which parts of the repository Anne is allowed to access, which are the obligations of Anne concerning the publication of knowledge items received as result of an interaction, and whether Anne allows for items that she provides to be published or not.

\subsubsection{Interaction Model}

When role enacting agents come together in an interaction scene, the actual interpretation of the scene script that is the interaction protocol to be used must be agreed upon. In OperA, role enacting agents will, for each scene, negotiate an interaction contract that defines their partnership, and fixes the way a specific interaction scene is to be played. Interaction contracts describe instances of scene scripts which inherit the organizational norms and objectives described in the interaction script and possibly extend or restrain it to accommodate the specific needs and desires of the participating agents.

A fictive but typically possible in the domain of non-life insurance contract between two members is, for example, the following. Anne will provide Bob with a report about competition prices, on the condition that Bob will give her comments on the report (that she will have to present to her Unit directors) and eventually share with her his new pricing concept for car insurance. This contract is generated during the 'Negotiate partnership' scene and registered in the 'Register partnership' scene. In this scene, the notary agent will assign a monitor agent to check the fulfillment of the contract between Anne and Bob.

\section{Conclusions}

Current developments in KM indicate a need for systems that are reactive and proactive in relation to the needs and expectations of its users. In this paper, we have discussed the role of agents in the design and functionality of knowledge management environments. In such environments, the flow of knowledge within an organisation (or organisations) must take in account not only the knowledge needs of business processes, but also the personal preferences and level of expertise of individual users.

Agent concepts, which originated in artificial intelligence but which have further developed and evolved in many areas of computing, hold great promise for responding to the new realities of knowledge management. While there are many conceptions of agents, most have embodied higher levels of representation and reasoning involving knowledge/belief, perception, goal, intention, and commitment. On the one hand, the technical embodiment of these concepts can lead to advanced functionality of KM systems, e.g. personalisation of knowledge presentation and 
matching supply and demand of knowledge. On the other, the rich representational capabilities of agents as modelling entities allow more faithful and effective treatments of complex organisational processes.

Although several projects are currently taking place that use agents for the design and implementation of KM systems, several research issues are still open that will provide a clear view of the applicability and consequences of using agents in KM. While the agent research area is very active, its concerns towards KM are not yet well covered. Agent concepts could fundamentally alter the nature of knowledge management both in the way KM systems are build as well as the way organisations are analysed and modelled.

Future research in agent-oriented approaches to knowledge management and collaborative systems must include:

- Methodologies are needed that support the analysis of knowledge management needs of organisations and its specification using software agents and agent societies

- Reusable agent-oriented knowledge management frameworks, including the description of agent roles, interaction forms and knowledge description

- Agent-based tools for organisational modelling and simulation that help determine the knowledge processes of the organisation,

- The role of learning in agent-based knowledge management systems, namely, how to use agent learning to support and extend knowledge sharing.

\section{References}

[1] V. Dignum: A Model for Organizational Interaction: Based on Agents, Founded in Logic. PhD thesis, Utrecht University, (2004).

[2] V. Dignum, F. Dignum: Modeling agent societies: coordination frameworks and institutions. In: Brazdil, P, Jorge, A.: Progress in Artificial Intelligence, LNAI 2258, Springer, pp. 191 - 204, 2001.

[3] V. Dignum, F. Dignum, J.J. Meyer: An Agent-Mediated Approach to the Support of Knowledge Sharing in Organizations. Knowledge Engineering Review, Cambridge University Press, 19(2), pp. 147-174, 2004.

[4] L. van Elst, V. Dignum, A. Abecker: Towards Agent-Mediated Knowledge Management. In: L. van Elst, V. Dignum, A. Abecker (Eds): Agent-Mediated Knowledge Management: Selected Papers, LNAI 2926, Springer, 2004.

[5] J. Ferber. Multi-Agent System: An Introduction to Distributed Artificial Intelligence. Harlow: Addison Wesley Longman. 1999.

[6] Jennings, N., Faratin, P., Johnson, M., Norman, T., O'Brien, P., \& Wiegand M.: Agentbased business process management. International Journal of Cooperative Information Systems, 5(2\&3), pp. $105-130,1996$.

[7] N. Jennings \& M. Wooldridge: Applications of Intelligent Agents. In: Jennings, N. and Wooldridge, M. (Eds.): Agent Technology: Foundations, Applications and Markets, Springer, 1998, 3 - 28.

[8] Klusch, M. (Ed.): Intelligent Information Agents: Agent-based Information Discovery and Management in the Internet. Springer, 1999.

[9] Koch, M.: Knowledge Management and Knowledge Agents in Campiello. In: Lees, B., Müller, H., Branki, C. (Eds.): Proc. Workshop on Intelligent Agents in CSCW, Dortmund, Germany, pp. 44-52, 1998. 
[10] O. Kühn and A. Abecker. Corporate memories for knowledge management in industrial practice: Prospects and challenges. In U.M. Borghoff and R. Pareschi (Eds): Information Technology for Knowledge Management. Springer, 1998.

[11] Leake, D., Birnbaum, L., Hammond, K., Marlow, C. \& Yang, H.: Task-Based Knowledge Management. In: Proc. AAAI Workshop Exploring Synergies of Knowledge Management and Case-Based Reasoning, AAAI Technical Report WS-99-10, 1999.

[12] H. Lieberman. Letizia: An agent that assists web browsing. In C. Mellish (Ed):, Proc. IJCAI-95, Montreal, Canada, August 1995.

[13] P. Maes. Agents that Reduce Work and Information Overload. In Communications of the ACM, 37(7), July 1994

[14] G. Mentzas, D. Apostolou, R. Young, and A. Abecker. Knowledge Asset Management: Beyond the Process-centred and Product-centred Approaches. Advanced Information and Knowledge Processing. Springer-Verlag, 2002.

[15] Preece, A., Hui, K.-Y., Gray, W., Marti, P., Bench-Capon, T. Jones D. \& Cui Z.: The KRAFT Architecture for Knowledge Fusion and Transformation. 19th SGES International Conference on Knowledge-based Systems and Applied Artificial Intelligence, 1999.

[16] S. Staab and H.-P. Schnurr. Smart task support through proactive access to organizational memory. Knowledge-based Systems, 13(5): 251-260, 2000.

[17] J. Vazquez-Salceda, V. Dignum, F. Dignum: Organizing Multiagent Systems. JAAMAS, Springer, online 29 June, 2005.

[18] F. Zambonelli, N. Jennings, M. Wooldridge: Organizational Abstractions for the Analysis and Design of Multi-Agent Systems. In: P. Ciancarini, M. Wooldridge (eds.): Agent-Oriented Software Engineering. LNAI 1957. Springer, 2001. 